\title{
Weniger Schmerzen durch virtuelle Realität
}

— Beim Arztbesuch virtuelle Pinguine beim Schwimmen beobachten oder den Mount Everest besteigen - ein Ausflug in die virtuelle Realität kann Patienten von einer schmerzhaften Behandlung ablenken. Nun stellten Forscher um Birgit Nierula von der Universität Barcelona in einer Online-Publikation der Fachzeitschrift "Journal of Pain" einen neuen Ansatz vor, der Patienten mithilfe von Virtual-Reality-Brillen helfen kann, Schmerzen weniger stark zu spüren. Der Effekt ist allerdings überschaubar.

Bereits seit Jahren ist bekannt, dass Menschen weniger empfindlich sind, wenn sie während einer schmerzhaften Behandlung auf ihren Körper sehen. Die Neurowissenschaftler um Nierula prüften nun, ob dieser Effekt auch mithilfe virtueller Realität funktioniert. Dabei nutzten sie ein psychologisches Phänomen: Bei der sogenannten Gummihand-Illusion nehmen Menschen unter bestimmten Umständen eine unechte Hand als Teil ihres Körpers wahr.

Die Forscher ließen 19 Freiwillige zunächst auf einem Stuhl sitzen und setzten ihnen eine Virtual-Reality-Brille auf. Durch diese sah jeder Proband eine Person, die in der gleichen Position auf einem Stuhl saß wie er selbst. Sie hielt einen Knopf in der linken Hand, genau wie der Teilnehmer. Wenn nun der virtuelle Knopf vibrierte, spürte der Teilnehmer eine Vibration in der eigenen Hand. So sollte er die virtuelle Hand als Teil des eigenen Körpers empfinden. Im nächsten Schritt untersuchten die Forscher, ob die Freiwilligen unempfindlicher gegenüber Schmerzen waren, wenn sie auf das virtuelle Körperteil sahen. Die Teilnehmer nahmen Elektroden in die rechte Hand, die sich langsam aufheizten. Sie mussten beurteilen, ab wann die Hitze schmerzhaft wurde.

Das Resultat: Wenn die Freiwilligen die virtuelle Hand als Teil ihres Körpers wahrnahmen, waren sie weniger schmerzempfindlich. Sie meldeten erst ab durchschnittlich $45,2^{\circ} \mathrm{C}$, dass ihnen die Elektrode zu heiß wurde. Den anderen Teilnehmern wurde der Schmerz bereits ab $44,7^{\circ} \mathrm{C}$ zu viel.

(dpa)

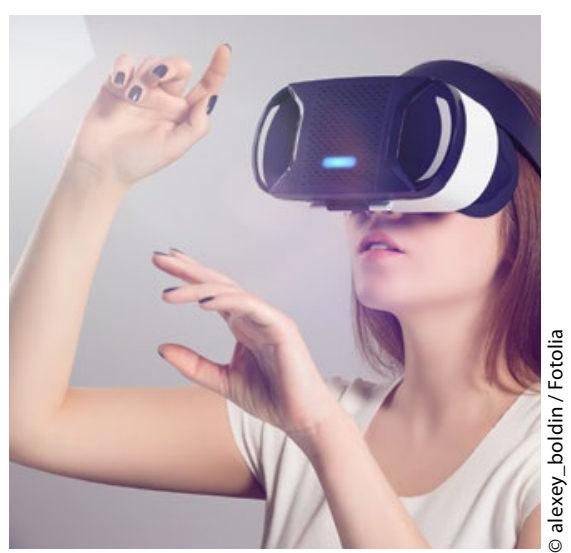

Prophylaxeerfolg

\section{Elektroakupunktur} punktet bei Migräne

— Mithilfe der Elektroakupunktur über vier Wochen gelingt es bei Patienten mit Migräne ohne Aura, die Häufigkeit der Attacken langanhaltend zu senken. Zusätzlich wird die Migränedauer verkürzt. Dies geht aus einer randomisierten Studie aus China hervor (Zhao et al. JAMA Intern Med 2017 Feb 20). Insgesamt 242 Patienten mit Migräne ohne Aura im Alter zwischen 18 und 65 Jahren nahmen an der Studie teil. Die Akupunktur erfolgte innerhalb von vier Wochen an jeweils fünf Tagen pro Woche. Hierfür verwendeten die Mediziner das HANS $^{\circledR}$ System des Han-Instituts in Nanjing. Die Stimulationsfrequenz betrug $2 / 100 \mathrm{~Hz}$. Pro Sitzung wurden vier Akupunkturpunkte stimuliert. Dazu gehörten immer die zwei Punkte GB20 (im Nacken auf der Haargrenze in Höhe des Ohrläppchens) und GB8 (oberhalb des äußeren Ohres). Zur Auswahl standen zudem die Punkte SJ5, GB34, BL60, SI3, LI4, ST44, LR3 und GB40. In der Kontrollgruppe wurden Punkte gewählt, die nicht als klassische Akupunkturpunkte gelten.

Die Häufigkeit der Migräneattacken nahm in der Gruppe mit korrekter Akupunktur um 3,2 ab, in der Gruppe mit Scheinakupunktur dagegen nur um 2,1 (95\%-Konfidenzintervall: $0,4-1,9 ; p<0,002$ ). Die Zahl der Migränetage war in der Verumgruppe ebenfalls signifikant niedriger als in der Gruppe mit Scheinakupunktur.

(ple)

\section{Neuralgien}

\section{Nervenschmerzen wirksam und dosiert behandeln}

— Für Betroffene mit Trigeminusneuralgie gibt es Hoffnung - mit einem neuen Wirkstoff können die Schmerzen auf ein erträgliches Niveau gesenkt werden. Dies zeigen Resultate einer internationalen Phase-II-Studie unter Beteiligung des Zentrums für Zahnmedizin der Universität Zürich (Zakrzewska JM et al. Lancet Neurol 2017 Feb 16). Schmerzsignale erreichen das Hirn über die Aktivierung von
Natriumkanälen, die in der Membran von Nervenzellen eingelagert sind. Der Natriumkanal 1.7 kommt häufig bei schmerzleitenden Nerven vor und ist umso aktiver, je stärker die Schmerzen sind. Eine Blockade dieses Natriumkanals - etwa mittels einer Lokalanästhesie - könnte den Schmerz stoppen. Doch da der Nervenschaden bei der Trigeminusneuralgie an der Schädelbasis vermutet wird, kann er nicht mit einer lokalen Injektion therapiert werden, sondern erfordert eine medikamentöse Behandlung.

Das in der schweizerischen Studie getestete Medikament mit dem Wirkstoff BIIB074 hemmt den Natriumkanal 1.7 und zwar in Abhängigkeit von dessen Aktivitätszustand: Je aktiver dieser Natriumkanal ist, desto stärker wird er durch BIIB074 gehemmt. Die bisher verwendeten Wirkstoffe hatten im Gegesatz dazu den Natriumkanal 1.7 unabhängig von der Nervenaktivität blockiert, was mit belastenden Nebenwirkungen einhergegangen war.

(red) 